\title{
Preliminary Report on the Flora of Southern Hidaka, Hokkaido (Yezo). XXX.
}

\author{
By
}

Hiroshi Hara.

Received January 18, 1938.

\section{Cyperaceae.}

Carex L.

684 ${ }^{(1)}$ \%*arex aphanolepis Franchet et Savatier, Enum. Pl. Jap. II, p. 152 (1876) nom. seminud. \& p. 580 (1877).-Franchet in Bull. Soc. Philom. Paris ser. 8, VII, p. 89 (1895).-Mryabe et Kudo, Fl. Hokk. \& Saghal. II, p. 263 (1931).-Akiyama, Consp. Car. Jap. in Journ. Fac. Sei. Hokkaido Imp. Univ. ser. V, II-1, p. 195, f. 140 (1932).-Orw I, Cyperac. Jap. I, Synop. Car. Jap. in Mem. Coll. Sci. Kyoto Imp. Univ. ser. B, XI-5, p. 454 (1936).

Nom. Jap. Enashi-higokusa, Sawasuge.

Hab. at the foot of Mt. Apoi, Nikanbetsu valley, etc. Fl. Jun.

Dist. Kyushu, Shikoku, Honshu, Yezo and Korea.

685(2)*Carex blepharicarpa Franchet in Bull. Soc. Philom. Paris ser. 8, VII, p. 46 (1895).-Tatew., Veg. Apoi p. 118 (1928).-Nakai, Veg. Apoi p. 75 (1930).-Miyabe et Kudo, Fl. Hokk. \& Saghal. II, p. 249 (1931).-Akiyama, Consp. Car. Jap. p. 134, f. 82 (1932).-OHwi, Synop. Car. Jap. p. 336 (1936).

Nom. Jap. Shôjố-suge.

Hab. in Mt. Apoi (ex Tatewaki \& Nakai).

Dist. Kyushu, Shikoku, Honshu, Yezo, Kuriles, Saghalien and Kamtchatka.

686 (3) Carex capillacea Bоотт, Ill. I, p. 44, t. 110 (1858).

var. sachalinensis OHwi, Synop. Car. Jap. p. 442 (1936).

Syn. Carex nana (non Lamarck 1789, nec Chamisso 1855) Boott in A. Gray, Bot. Jap. p. 418 (1859).-Aкiyama, Consp. Car. Jap. p. 55, f. 8 (1932).

Carex uda var. sachalinensis Fr. Schmid, Reis. Amur. u. Sachal. p. 191 (1868).

Carex aomorensis Franchet, Carex As.-Or. p. 198 (1896).

Nom. Jap. Michinoku-harisuge. 
Hab. in Mt. Horoman. Fl. Jun.

Dist. Honshu, Yezo, Kuriles, Saghalien and Korea.

687(4)***arex confertiflora Boотт in A. Gray, Bot. Jap. p. 418 (1859).Mryabe et Kudo, Fl. Hokk. \& Saghal. II, p. 262 (1931).-Akiyama, Consp. Car. Jap. p. 191, f. 137 (1932).-OHwi, Synop. Car. Jap. p. 476 (1936).

Nom. Jap. Miyama-shirasuge (Makino).

Hab. in marshy places at the foot of Mt. Apoi. Fl. Jul.

Dist. Kyushu, Shikoku, Honshu and Yezo.

688 ${ }^{(5)}$ ***arex forficula Franchet et Savatier, Enum. Pl. Jap. II, p. 131 (1876) nom. nud. \& p. 557 (1877).-Mryabe et Kudo, Fl. Hokk. \& Saghal. II, p. 235 (1931).-Акiуnмa, Consp. Car. Jap. p. 95, f. 44 (1932).-Онwi, Synop. Car. Jap. p. 291 (1936).

Nom. Jap. Tanigawa-suge (Makino).

Hab. at the foot of Mt. Apoi. Fl. May-Jun.

Dist. Kyushu, Shikoku, Honshu, Yezo, Korea, China, Manchuria and Ussuri.

689 ${ }^{(6)}$ *Carex Gmelini Hooker et Arnotr, Bot. Beechey Voy. p. 118, t. 27 (1832).-Franchet in Bull. Soc. Philom. Paris ser. 8, VII, p. 36 (1895).Mryabe et Kudo, Fl. Hokk. \& Saghal. II, p. 241 (1931).-Akiyama, Consp. Car. Jap. p. 115, f. 64 (1932).-OHwi, Synop. Car. Jap. p. 318 (1936).

Syn. Carex flavocuspis (non Franchet et Savatier) Nakai, Veg. Apoi p. 75 (1930).

Nom. Jap. Nemuro-suge.

Hab. in coastal places at the foot of Mt. Apoi, C. Erimo, Saruru, etc. Fl. Jun.

Dist. Yezo, Kuriles, Saghalien, Korea, Manchuria, Ussuri, Amur, Ochotsk, Anadyr, Kamtchatka and Alaska.

$690^{(7)}$ Carex hakonensis Franchet et Savatier, Enum. Pl. Jap. II, p. 123 (1876) nom. seminud. \& p. 550 (1877).-Mryabe et Kudo, Fl. Hokk. \& Saghal. II, p. 215 (1931).-Aкiуama, Consp. Car. Jap. p. 53 (1932).-Оhwi, Synop. Car. Jap. p. 437 (1936).

Syn. Carex Krameri Franchet et Savatier, l. c. p. 124 \& 550.-Akiyama, l. c. f. 7 (1932).

Nom. Jap. Ko-harisuge.

Hab. near Shoya. Fl. May-Jun.

Dist. Kyushu, Shikoku, Honshu, Yezo and Korea.

$691^{(8) *}$ Carex humilis Leysser, Fl. Halen. p. 175 (1761).-Akiyama, Consp. Car. Jap. p. 158 (1932).-OHwi, Synop. Car. Jap. p. 399 (1936). 
*var. nana OHwi, l. c. in descrip. (1936).

Syn. Carex lanceolata var. nana Léveillé et Vaniot in Bull. Acad. Intern. Geogr. Bot. X, p. 269 (1901).

Carex nanella OHwi in Mem. Coll. Sci. Kyoto Imp. Univ. ser. B, V, p.263(1930).-Miyabe et Kudo, Fl. Hokk. \& Saghal. II, p. 254(1931). Carex tenuissima (non Boотt) NakaI, Veg. Apoi p. 24 \& 75 (1930).-Miyabe et Kudo, l. c. p. 251 (1931).

Nom. Jap. Hosoba-hikagesuge.

Hab. in Mt. Apoi, Samani, ete. Fl. May.

Dist. Kyushu, Shikoku, Honshu, Yezo, S. Kuriles, Korea, Manchuria, Ussuri and Dahuria.

$692^{(9)}$ Carex incisa Boott in A. Gray, Perry's Exped. Jap. II, p. 327 (1857).-Miyabe et Kudo, Fl. Hokk. \& Saghal. II, p. 235 (1931).-Aкiyama, Consp. Car. Jap. p. 98 (1932).-Oнwi, Syn. Car. Jap. p. 279 (1936).

Nom. Jap. Kawara-suge.

Hab. in Mt. Horoman. Fl. Jun.

Dist. Honshu and Yezo.

693(10)*Carex japonica Thunbeig, Fl. Jap. p. 38 (1784).-Nakai, Veg. Apoi p. $24 \& 75$ (1930).-Miyabe et Kudo, Fl. Hokk. \& Saghal. II, p. 262 (1931).-Akiyama, Consp. Car. Jap. p. 194, f. 139 (1932).-OHwi, Synop. Car. Jap. p. 453 (1936).

Nom. Jap. Higokusa.

Hab. at the foot of Mt. Apoi. Fl. Jun.

Dist. Kyushu, Shikoku, Honshu, Yezo, Korea, Manchuria and China.

694(11)*Carex lanceolata Boоt' in A. Gray, Perry's Exped. Jap. II, p. 326 (1857).-Nakar, Veg. Apoi p. 75 (1930).-Mryabe et Kudo, Fl. Hokk. \& Saghal. II, p. 253 (1931).-Akiyama, Consp. Car. Jap. p. 156, f. 104 (1932). -Oнwi, Synop. Car. Jap. p. 401 (1936).

Nom. Jap. Hiliage-suge.

Hab. at the foot of Mt. Apoi. Fl. May-Jun.

Dist. Kyushu, Shikoku, Honshu, Yezo, Korea, China, Manchuria, Ussuri, Amur and Siberia.

695(12)*Carex lasiolepis Franchet in Bull. Soc. Philom. Paris ser. 8, VII, p. 46 (1895).--Tatew., Veg. Apoi p. 118 (1928).-Nakai, Veg. Apoi p. 75 (1930).-Mryabe et Kudo, Fl. Hokk. \& Saghal. II, p. 253 (1931).-Akryama, Consp. Car. Jap. p. 155, f. 103 (1932).-OHwi, Synop. Car. Jap. p. 394 (1936). Syn. Carex adumana Makino in Bot. Mag. Tokyo IX, p. [258] (Jul. 1895).

Nom. Jap. Adzuma-suge.

Hab. at the foot of Mt. Apoi. 
Dist. Honshu, Yezo and Korea.

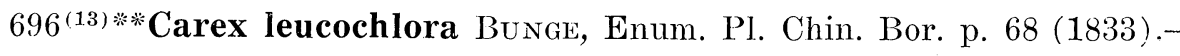
Akiyama in Journ. Jap. Bot. XIII, p. 653 (1937).

Syn. Carex Royleana Nees in Wight, Contr. Bot. Ind. p. 127 (1834).Miyabe et Kudo, Fl. Hokk. \& Saghal. II, p. 250 (1931).-Akiyama, Consp. Car. Jap. p. 135, f. 83 (1932).

Carex breviculmis (non R. Brown) OHwi, Synop. Car. Jap. p. 353 (1936) pro parte.

Nom. Jap. Awo-suge.

Hab. at the foot of Mt. Apoi. Fl. May.

Dist. Formosa, Liukiu, Kyushu, Shikoku, Honshu, Yezo, Korea, Manchuria, Ussuri, Amur, China and India.

697(14)*Carex Maximowiczii Miquel in Ann. Mus. Bot. Lugd.-Bat. II, p. 150 (1865).-Nakai, Veg. Apoi p. 75 (1930).-Mryabe et Kudo, Fl. Hokk. \& Saghal. II, p. 236 (1931).-Akryama, Consp. Car. Jap. p. 99, f. 48 (1932).Oнw, Synop. Car. Jap. p. 292 (1936).

Nom. Jap. Gôso.

Hab. at the foot of Mt. Apoi. Fl. Jul.

Dist. Liukiu, Kyushu, Shikoku, Honshu, Yezo, S. Kuriles, Korea, China and Manchuria.

698 (15) Carex Myabei Franchet in Bull. Soc. Philom. Paris. ser. 8, VII, p. 52 (1895).--Miyabe et Kudo, Fl. Hokk. \& Saghal. II, p. 275 (1931).-Akiyama, Consp. Car. Jap. p. 233, f. 171 (1932).-OHwi, Synop. Car. Jap. p. 508 (1936).

Nom. Jap. Birôdo-suge.

Hab. between Unbe and Utoma. Fl. Aug.

Dist. Kyushu, Honshu and Yezo.

699(16)*Carex mollicula Boоtт, Ill. IV, p. 192 (1867).-Franchet in Bull. Soc. Philom. Paris ser. 8, VII, p. 49 (1895).-Tatew., Veg. Apoi p. 118 (1928).-Nakai, Veg. Apoi p. 75 (1930).-Miyabe et Kudo, Fl. Hokk. \& Saghal. II, p. 263 (1931).-Aкiyama, Consp. Car. Jap. p. 197, f. 143 (1932). -OHwi, Synop. Car. Jap. p. 457 (1936).

Nom. Jap. Hime-shirasuge.

Hab. at the foot of Mt. Apoi, Saruru, etc. Fl. Jun.-Jul.

Dist. Kyushu, Shikoku, Honshu, Yezo, S. Kuriles, Saghalien, S. Korea and China.

700 (17) Carex Oederi Retzius, Fl. Scand. Prodr. p. 179 (1779).-Franchet in Bull. Soc. Philom. Paris ser. 8, VII, p. 49 (1895).-OHwi, Synop. Car. 
Jap. p. 479 (1936).

var. viridula KüKENTHAL in ENGLER, Pfl.-reich. IV-20, p. 674 (1909). -Miyabe et Kudo, Fl. Hokk. \& Saghal. II, p. 268 (1931).-Airiyama, Consp. Car. Jap. p. 217, f. 160 (1932).

Syn. Carex viridula Michaux, Fl. Bor. Amer. II, p. 170 (1803).-KreczeTOVICZ in Fl. URSS. III, p. 389 (1935).

Nom. Jap. Yezo-sawasuge.

Hab. in Mt. Horoman. Fl. Jun.

Dist. Honshu, Yezo, Kuriles, Saghalien, Kamtchatka and N. America.

$701^{(18)}$ Carex oxyandra Kudo, Rep. Veg. N. Saghal. p. 72 (1924).-Mryabe et Kudo, Fl. Hokk. \& Saghal. II, p. 246 (1931).-Akıyama, Consp. Car. Jap. p. 127, f. 74 (1932).-Oнwi, Synop. Car. Jap. p. 410 (1936).

Syn. Carex montana var. oxyandra Franchet et Savatier, Enum. Pl. Jap. II, p. 141 (1876) \& p. 565 (1877).

Carex Wrightii (non Dewey 1858) Franchet in Bull. Soc. Philom. Paris ser. 8, VII, p. 47 (1895).

Nom. Jap. Hime-suge.

Hab. on Mt. Rakko. Fl. Jun.

Dist. Formosa, Kyushu, Shikoku, Honshu, Yezo, Kuriles and Saghalien.

$702^{(19)}$ Carex pumila Thunberg, Fl. Jap. p. 39 (1784).-Mryabe et Kudo, Fl. Hokk. \& Saghal. II, p. 273 (1931).-Aкгyama, Consp. Car. Jap. p. 228, f. 168 (1932).-Онwi, Synop. Car. Jap. p. 485 (1936).

Nom. Jap. Kôbôshiba.

Hab. in coastal sandy places of C. Erimo. Fl. Jun.

Dist. Formosa, Liukiu, Kyushu, Shikoku, Honshu, Yezo, S. Kuriles, Saghalien, Korea, China, Manchuria and Ussuri.

$703^{(20)}$ * Carex sabynensis Lessing ex Kunth, Enum. Pl. II, p. 440 (1837). -Miyabe et Kudo, Fl. Ilokk. \& Saghal. II, p. 249 (1931).-Akiyama, Consp. Car. Jap. p. 133, f. 81 (1932).--KREczetovicz in Fl. URSS. III, p. 329 (1935). -OHwi, Synop. Car. Jap. p. 352 (1936).

Nom. Jap. Kamikawa-suge.

Hab. at the foot of Mt. Apoi. Fl. May.

Dist. Honshu, Yezo, S. Kuriles, Saghalien, Korea, Manchuria, Mongolia, Amur, Ochotsk, Dahuria and Siberia.

$704^{(21)}$ Carex scita Maximowicz in Bull. Acad. Imp. Sci. St.-Pétersb. XXXI, p. 115 (1887).

var. riishirensis Kükenthal in Engler, Pfl.-reich IV-20, p. 414 (1909).-Miyabe et Kudo, Fl. Hokk. \& Saghal. II, 245 (1931).-Akiyama, Consp. Car. Jap. p. 124 (1932).-OHwi, Synop. Car. Jap. p. 321 (1936). 
Syn. Carex riishirensis Franchet in Bull. Soc. Philom. Paris ser. 8, VII, p. 88 (1895).

Nom. Jap. Riishiri-suge, Mashike-suge.

Hab. on Mt. Rakko \& Mt. Tokachi. Fl. Jul.

Dist. Yezo and Saghalien.

$705^{(22) *}$ Carex siderosticta Hance in Journ. Linn. Soc. XIII, p. 89 (1873). -Tatew., Veg. Apoi p. 118 (1928) (var. bracteosa Fr.).-Nakai, Veg. Apoi p. 75 (1930).-Miyabe et Kudo, Fl. Hokk. \& Saghal. II, p. 256 (1931).-AkIyama, Consp. Car. Jap. p. 164 (1932).-OHwi, Synop. Car. Jap. p. 430 (1936).

\section{Nom. Jap. Taganesô.}

Hab. in Mt. Apoi, Samani, etc. Fl. May-Jun.

Dist. Kyushu, Shikoku, Honshu, Yezo, Korea, China and Manchuria.

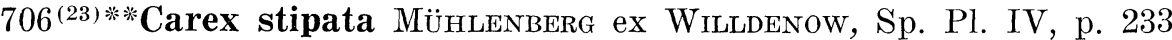
(1805).-Miyabe et Kudo, Fl. Hokk. \& Sahal. II, p. 220 (1931).-Akiyama, Consp. Car. Jap. p. 65, f. 18 (1932).-OHwi, Synop. Car. Jap. p. 245 (1936).

Nom. Jap. O-kawadzusuge.

Hab. at the foot of Mt. Apoi, Mt. Ruchishi, etc. Fl. Jun.

Dist. Honshu, Yezo, Ussuri and N. America.

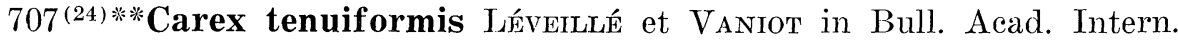
Géogr. Bot. XI, p. 104 (1902).-Miyabe et Kudo, Fl. Hokk. \& Saghal. II, p. 259 (1931).-Oнwi, Synop. Car. Jap. p. 448 (1936).

Syn. Carex koreana (non C. coreana Bailey 1889) Komarov in Acta Hort. Petrop. XVIII, p. 446 (1901).-Akiyama, Consp. Car. Jap. p. 179, f. 125 (1932).

Nom. Jap. Onoe-suge (Matsumura).

Hab. in the upper part of Mt. Apoi. Fl. Jun.

Dist. Mid. \& N. Honshu, Yezo, S. Kuriles, Saghalien and Korea.

708 ${ }^{(25) * *}$ Carex Thunbergii STEUdeL in Flora XXIX, p. 23 (1846).-MIYABE et Kudo, Fl. Hokk. \& Saghal. II, p. 231 (1931).-Akixama, Consp. Car. Jap. p. 87, f. 38 (1932).-Oнwi, Synop. Car. Jap. p. 280 (1936).

Nom. Jap. Aze-suge.

Hab. at the foot of Mt. Apoi. Fl. Jun.

Dist. sp. Shikoku, Honshu, Yezo, S. Kuriles, Saghalien, Korea, China, Manchuria, Ussuri, Amur, Ochotsk, Kamtchatka and Siberia.

$709^{(26)}$ Carex urostachys Franchet in Bull. Soc. Philom. Paris ser. 8, VII, p. 35 (1895).-OHwi, Synop. Car. Jap. p. 315 (1936).

Syn. Carex Mertensii var. urostachys KüKenthal in Engler, Pfl.-reich 
IV-20, p. 401 (1909).-Mryabe et Kudo, Fl. Hokk. \& Saghal. II, p. 242 (1931).-Akiyama, Consp. Car. Jap. p. 118, f. 66 (1932).

Nom. Jap. Kinchaku-suge.

Hab. on Mt. Rakko. Fl. Jun.

Dist. Honshu, Yezo and Kuriles.

Cyperus L.

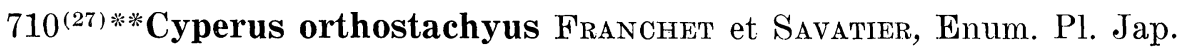
II, p. 106 (1876) nom. nud. \& p. 539 (1877).-HARA in Journ. Jap. Bot. XIV, p. 338 (1938).

Syn. Cyperus truncatus Turcz. var. orthostachyus Clakke in Journ. Linn.

Soc. XXXVI, p. 218 (1903).-Matsumurs, Ind. Pl. Jap. II-1, p.

143 (1905) pro parte.-NakaI in Bot. Mag. Tokyo XLVII, p. 241

(1933).-Masamune, Fl. \& Geobot. Stud. Yakusima p. 512 (1934).-

Kükenthal in Engler, Pfl.-reich IV-20, Heft 101, p. 155 (1935).

Nom. Jap. Ko-ushikugu (Makino).

Hab. in humid places at the foot of Mt. Apoi, Shoya, Horoidzumi, etc. Fl. mid. Aug.-_Sep.

Dist. sp. Kyushu, Shikoku, Honshu, Yezo, Korea, China, Manchuria, Ussuri, Amur and Dahuria.

Fimbristylis VAHL.

711(28)*Fimbristylis subbispicata NeEs et Meyen in Nova Acta Acad. Leop.-Carol. Nat. Cur. XIX, Suppl. I, p. 75 (1843).-Matsumura, Ind. Pl. Jap. II-1, p. 152 (1905).-Tatew., Veg. Apoi p. 118 (1928).-Nakai, Veg. Apoi p. 75 (1930).-Miyabe et Kudo, Fl. Hokk. \& Saghal. II, p. 211 (1931).Masamune, Fl. \& Geobot. Stud. Yakusima p. 519 (1934).-Merrill in Journ. Arnold Arbor. XVIII, p. 61 (1937).

Syn. Fimbristylis japonica Siebold et Zuccarini in Zollinger, Syst. Verz. Ind. Jap. I, p. 62 (1854) nom. nud.

Nom. Jap. Yama-i.

Hab. at the foot of Mt. Apoi, ete. Fl. Aug.

Dist. Formosa, Liukiu, Kyushu, Shikoku, Honshu, Yezo, Korea, Manchuria, China, Malaysia and India.

Heleocharis R. BR. corr. Lestib. (nom. conserv.)

$712^{(29) * H e l e o c h a r i s ~ j a p o n i c a ~ M i q u e s ~ i n ~ A n n . ~ M u s . ~ B o t . ~ L u g d .-B a t . ~ I I, ~ p . ~}$ 142 (1865) (ut Elaeocharis).-Matsumura, Ind. Pl. Jap. II-1, p. 145 (1905). -Tatew., Veg. Apoi p. 118 (1928).-Nakai, Veg. Apoi p. 75 (1930).-Mryabe et Kudo, Fl. Hokk. \& Saghal. II, p. 210 (1931).-Masamune, Fl. \& Geobot. Stud. Yakusima p. 516 (1934). 
Syn. Scirpus japonicus Franchet et Savatier, Enum. Pl. Jap. Il, p. 109 (1876).

Nom. Jap. Hari-i.

Hab. at the foot of Mt. Apoi. Fl. Jul.

**var. thermalis (HuLtéN) HaRA, comb. nov.

Syn. Scirpus ,japonicus var. thermalis Hultén, Fl. Kamt. I, p. 165, pl. 5, e (1927).

Heleocharis Maximowiczii ZINSERLING in Fl. URSS. III, p. 88 \& 588 (1935).

Nom. Jap. Yezo-harii (nov.).

Hab. at the foot of Mt. Apoi. Fl. Jul.

Dist. sp. Formosa, Liukiu, Kyushu, Shikoku, Honshu, Yezo, Korea, Manchuria, Ussuri, Amur, Kamtehatka, China and Malaysia.

This form has a little larger achene with broader deltoid stylopodium than the typical $H$. japonica. According to ZinserLing who examined the cotype specimen of H.afflata Steudel (1855) in Leningrad, it is identical with $H$. japonica, while HANDEL-MAzzeTtr regards it to be conspecific with H. pellucida PresL (1830).

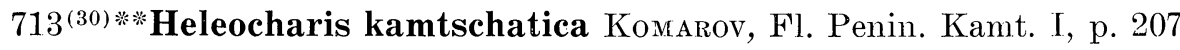
(1.927) (ut Eleocharis).-Fernald et BRAcketT in Rhodora 31, p. 75, pl. 184, f. 32-33 (1929).-Mryabe et Kudo, Fl. Hokk. \& Saghal. II, p. 209 (1931).Ohiwi, in Bot. Mag. Tokyo XLV, p. 184 (1931).-Zinserling in Fl. URSS. III, p. 81 (1935).

Syn. Scirpus kamtschaticus C. A. Meyer in Mém. Acad. St.-Pétersb. Sav. Etr. I, p. 198 (1831).

Scirpus sachalinensis Meinshausen in Acta Hort. Petrop. XVIII, p. 260 (1901).

Eleocharis Savatieri Clarke ex LÉveillé in Bull. Int. Geogr. Bot. XIV, p. 203 (1904) nom. nud.-Matsumura, Ind. Pl. Jap. II-1, p. 146 (1905) pro parte-Clarke in Bull. Misc. Inform. Kew add. ser., VIII, p. 21 (1908) pro parte.

Eleocharis sachalinensis Komarov et Klob.-Alisova, Key Pl. Far East.

Reg. URSS. I, p. 266, t. 79, f. 1, 2, 4, 8, 9 (1931).-Zinserling, l. e. p. 80 (1935).

Nom. Jap. Hime-numaharii.

Hab. in marshy places near Fuyushima, Shoya, etc. Fl. Jul.

Dist. Honshu, Yezo, Kuriles, Saghalien, Ussuri, Ochotsk, Kamtchatka, Aleutian and Alaska. 